\title{
SU(2) gauge theory of the Hubbard model: Emergence of an anomalous metallic phase near the Mott critical point
}

\author{
Ki-Seok Kim \\ School of Physics, Korea Institute for Advanced Study, Seoul 130-012, Korea
}

(Dated: September 16, 2018)

\begin{abstract}
We propose one possible mechanism for an anomalous metallic phase appearing frequently in two spatial dimensions, that is, local pairing fluctuations. Introducing a pair-rotor representation to decompose bare electrons into collective pairing excitations and renormalized electrons, we derive an $\mathrm{SU}(2)$ gauge theory of the Hubbard model as an extended version of its $\mathrm{U}(1)$ gauge theory[1, $2]$ to allow only local density fluctuations. Since our effective $\mathrm{SU}(2)$ gauge theory admits two kinds of collective bosons corresponding to pair excitations and density fluctuations respectively, an intermediate phase appears naturally between the spin liquid Mott insulator and Fermi liquid metal of the $\mathrm{U}(1)$ gauge theory, $[1,2]$ characterized by softening of density-fluctuation modes as the Fermi liquid, but gapping of pair-excitation modes. We show that this intermediate phase is identified with an anomalous metallic phase because there are no electron-like quasiparticles although it is metallic.
\end{abstract}

PACS numbers: 71.10.Hf, 71.30.+h, 71.10.-w, 71.10.Fd

It has been a common belief for a long time in the condensed matter community that a metallic phase does not exist at zero temperature in two spatial dimensions owing to localization by disorder, even if electron-electron interactions exist. [3, 4] However, if one considers a non-Fermi liquid phase instead of a Fermi liquid state, characterized by the absence of electron quasiparticles due to an anomalous exponent in the electron spectral function, he can show that disorder-induced interactions in the replica formalism are irrelevant for moderate values of the exponent in the renormalization group sense. $[5,6]$ In this paper we demonstrate how the non-Fermi liquid metal can arise from strong electron-electron interactions, based on the Hubbard model as a minimal model. We show that local pairing fluctuations can cause an anomalous metal, described by an effective U(1) gauge theory.

One may introduce a local pairing order parameter by utilizing a Hubbard-Stratonovich (HS) transformation. Integrating out gapless electrons, one obtains an effective action of the pairing order parameter with dissipation that results from the gapless electrons near the Fermi surface. It was conjectured that the presence of dissipation may result in a metallic phase in two dimensional interacting electrons. $[4,7]$ However, there are several unsatisfactory points in the order parameter approach. Since the gradient expansion used in this method is basically a weak coupling approach, the breakdown of this weak coupling approach is expected in a large coupling limit. In addition, it is difficult to justify the gradient expansion in the presence of gapless electrons because they can cause nonlocal interactions between order parameters, making it unreliable a conventional treatment in a local effective action.[8] Furthermore, the conjecture is not convincing yet that dissipation can give rise to a metallic phase in two dimensions.[4]

In this respect we utilize a strong coupling approach, that is, decomposing bare electrons $c_{\sigma}$ into collective excitations $U_{\sigma \sigma^{\prime}}$ and renormalized electrons $f_{\sigma}$, i.e., $c_{\sigma}=$
$U_{\sigma \sigma^{\prime}}^{\dagger} f_{\sigma^{\prime}} \cdot[9]$ Here local pairing excitations can be imposed in the $U_{\sigma \sigma^{\prime}}$ field. Recently, Florens and George proposed a slave-rotor representation of the Hubbard model, where the collective boson mode is set to be $U_{\sigma \sigma^{\prime}}=e^{i \theta} \delta_{\sigma \sigma^{\prime}}$, associated with local density fluctuations.[1] Using the slave-rotor representation, Lee and Lee constructed a $\mathrm{U}(1)$ gauge theory of the Hubbard model to study a spin liquid Mott insulator to Fermi liquid transition in triangular lattice.[2] In this paper we extends the U(1) gauge theory formulation by allowing local pairing fluctuations. Then, the collective boson field $U_{\sigma \sigma^{\prime}}$ is expressed as an $\mathrm{SU}(2)$ matrix field, involved with both density and pairing fluctuations. This leads us to construct an $\mathrm{SU}(2)$ gauge theory of the Hubbard model. Since our SU(2) gauge theory admits two kinds of collective bosons corresponding to density fluctuations and local pairing excitations respectively, an intermediate phase is generically allowed between the spin liquid Mott insulator and Fermi liquid metal of the slave-rotor $\mathrm{U}(1)$ gauge theory via $\mathrm{SU}(2)$ symmetry breaking due to hole doping. We show that this intermediate state is identified with an anomalous metallic phase in the respect that there are no electron-like quasiparticles although it is metallic.

We consider the Hubbard Hamiltonian

$$
H=-t \sum_{i j \sigma} c_{i \sigma}^{\dagger} c_{j \sigma}+\frac{3 u}{2} \sum_{i} c_{i \uparrow}^{\dagger} c_{i \uparrow} c_{i \downarrow}^{\dagger} c_{i \downarrow},
$$

where $t$ is a hopping integral of electrons, and $u$ strength of on-site Coulomb repulsions. The local interaction term can be decomposed into pairing and density channels in the following way

$$
\begin{aligned}
& \frac{3 u}{2} \sum_{i} c_{i \uparrow}^{\dagger} c_{i \uparrow} c_{i \downarrow}^{\dagger} c_{i \downarrow}=\frac{u}{2} \sum_{i} c_{i \uparrow}^{\dagger} c_{i \downarrow}^{\dagger} c_{i \downarrow} c_{i \uparrow} \\
& +\frac{u}{2} \sum_{i}\left(\sum_{\sigma} c_{i \sigma}^{\dagger} c_{i \sigma}-1\right)^{2}+\frac{u}{2}\left(\sum_{\sigma} c_{i \sigma}^{\dagger} c_{i \sigma}-1\right) .
\end{aligned}
$$

Performing the HS transformation for the pairing and 
density interaction channels, we find an effective Lagrangian in the Nambu-spinor representation

$$
\begin{aligned}
& Z=\int D\left[\psi_{i}, \psi_{i}^{\dagger}, \Phi_{i}^{R}, \Phi_{i}^{I}, \varphi_{i}\right] e^{-\int d \tau L} \\
& L=\sum_{i} \psi_{i}^{\dagger}\left(\partial_{\tau} \mathbf{I}-\mu \tau_{3}\right) \psi_{i}-t \sum_{\langle i j\rangle}\left(\psi_{i}^{\dagger} \tau_{3} \psi_{j}+H . c .\right) \\
& -i \sum_{i}\left(\Phi_{i}^{R} \psi_{i}^{\dagger} \tau_{1} \psi_{i}+\Phi_{i}^{I} \psi_{i}^{\dagger} \tau_{2} \psi_{i}+\varphi_{i} \psi_{i}^{\dagger} \tau_{3} \psi_{i}\right) \\
& +\frac{1}{2 u} \sum_{i}\left(\Phi_{i}^{R 2}+\Phi_{i}^{I 2}+\varphi_{i}^{2}\right) .
\end{aligned}
$$

Here $\psi_{i}$ is the Nambu spinor, given by $\psi_{i}=\left(\begin{array}{c}c_{i \uparrow} \\ c_{i \downarrow}^{\dagger}\end{array}\right) . \Phi_{i}^{R}$ and $\Phi_{i}^{I}$ are the real and imaginary parts of the superconducting order parameter respectively, and $\varphi_{i}$ an effective density potential. $\mu$ is an electron chemical potential which differs from its bare value $\mu_{b}$ as $\mu=\mu_{b}-u / 2$. Introducing a pseudospin vector $\vec{\Omega}_{i} \equiv\left(\Phi_{i}^{R}, \Phi_{i}^{I}, \varphi_{i}\right)$, one can express Eq. (2) in a compact form

$$
\begin{aligned}
& Z=\int D\left[\psi_{i}, \psi_{i}^{\dagger}, \vec{\Omega}_{i}\right] e^{-\int d \tau L} \\
& L=\sum_{i} \psi_{i}^{\dagger}\left(\partial_{\tau} \mathbf{I}-\mu \tau_{3}\right) \psi_{i}-t \sum_{\langle i j\rangle}\left(\psi_{i}^{\dagger} \tau_{3} \psi_{j}+H . c .\right) \\
& -i \sum_{i} \psi_{i}^{\dagger}\left(\vec{\Omega}_{i} \cdot \vec{\tau}\right) \psi_{i}+\frac{1}{4 u} \sum_{i} \operatorname{tr}\left(\vec{\Omega}_{i} \cdot \vec{\tau}\right)^{2} .
\end{aligned}
$$

Integrating over the pseudospin field $\vec{\Omega}_{i}$, Eq. (3) recovers the Hubbard model Eq. (1).

As discussed in the introduction, we disintegrate bare electrons into collective excitations and renormalized electrons in the following way

$$
\psi_{i}=e^{-i \phi_{1 i} \tau_{1}-i \phi_{2 i} \tau_{2}-i \phi_{3 i} \tau_{3}} \eta_{i} \equiv U_{i}^{\dagger} \eta_{i},
$$

where the two component spinor $\eta_{i}=\left(\begin{array}{c}\eta_{i+} \\ \eta_{i-}^{\dagger}\end{array}\right)$ can be considered to express renormalized electrons, and the $\mathrm{SU}(2)$ matrix field $U_{i}=\exp \left[i \sum_{k=1}^{3} \phi_{k i} \tau_{k}\right]$ collective bosons. Here $\exp \left[i \phi_{1 i} \tau_{1}\right]$ (or $\exp \left[i \phi_{2 i} \tau_{2}\right]$ ) can be interpreted as a creation operator of an electron pair since it mixes a particle with a hole while $\exp \left[i \phi_{3 i} \tau_{3}\right]$ is identified with a creation operator of an electron charge, corresponding to the rotor variable in the slave-rotor representation. [1, 10]

Inserting the decomposition Eq. (4) into the effective Lagrangian Eq. (3), we obtain

$$
\begin{aligned}
& Z=\int D\left[\eta_{i}, \eta_{i}^{\dagger}, U_{i}, \vec{\Omega}_{i}\right] e^{-\int d \tau L}, \\
& L=\sum_{i} \eta_{i}^{\dagger}\left(\partial_{\tau} \mathbf{I}+U_{i} \partial_{\tau} U_{i}^{\dagger}-\mu U_{i} \tau_{3} U_{i}^{\dagger}\right) \eta_{i} \\
& -t \sum_{\langle i j\rangle}\left(\eta_{i}^{\dagger} U_{i} \tau_{3} U_{j}^{\dagger} \eta_{j}+H . c .\right) \\
& -i \sum_{i} \eta_{i}^{\dagger} U_{i}\left(\vec{\Omega}_{i} \cdot \vec{\tau}\right) U_{i}^{\dagger} \eta_{i}+\frac{1}{4 u} \sum_{i} \operatorname{tr}\left(\vec{\Omega}_{i} \cdot \vec{\tau}\right)^{2} .
\end{aligned}
$$

Performing the gauge transformation $\vec{\Omega}_{i} \cdot \vec{\tau} \rightarrow U_{i}^{\dagger}\left(\vec{\Omega}_{i}\right.$. $\vec{\tau}) U_{i}$, and shifting $\vec{\Omega}_{i} \cdot \vec{\tau} \rightarrow \vec{\Omega}_{i} \cdot \vec{\tau}-i U_{i} \partial_{\tau} U_{i}^{\dagger}+i \mu U_{i} \tau_{3} U_{i}^{\dagger}$, Eq. (5) reads

$$
\begin{aligned}
& Z=\int D\left[\eta_{i}, \eta_{i}^{\dagger}, U_{i}, \vec{\Omega}_{i}\right] e^{-\int d \tau L} \\
& L=\sum_{i} \eta_{i}^{\dagger}\left(\partial_{\tau} \mathbf{I}-i \vec{\Omega}_{i} \cdot \vec{\tau}\right) \eta_{i}-t \sum_{\langle i j\rangle}\left(\eta_{i}^{\dagger} U_{i} \tau_{3} U_{j}^{\dagger} \eta_{j}+H . c .\right) \\
& +\frac{1}{4 u} \sum_{i} \operatorname{tr}\left(-i U_{i} \partial_{\tau} U_{i}^{\dagger}+\vec{\Omega}_{i} \cdot \vec{\tau}+i \mu U_{i} \tau_{3} U_{i}^{\dagger}\right)^{2}
\end{aligned}
$$

Using the HS transformation for the hopping term

$$
\begin{aligned}
& -t\left(\eta_{i \alpha}^{\dagger} U_{i \alpha \beta} \tau_{3 \beta \gamma} U_{j \gamma \delta}^{\dagger} \eta_{j \delta}+\text { H.c. }\right) \\
& \rightarrow t\left[F_{i j \alpha \delta} E_{i j \delta \alpha}^{\dagger}+E_{i j \alpha \delta} F_{i j \delta \alpha}^{\dagger}\right. \\
& \left.-\left(\eta_{i \alpha}^{\dagger} F_{i j \alpha \delta} \eta_{j \delta}+U_{i \alpha \beta} \tau_{3 \beta \gamma} U_{j \gamma \delta}^{\dagger} E_{i j \delta \alpha}^{\dagger}\right)-H . c .\right]
\end{aligned}
$$

we find an effective Lagrangian of the Hubbard model

$$
\begin{aligned}
& Z=\int D\left[\eta_{i}, \eta_{i}^{\dagger}, U_{i}, \vec{\Omega}_{i}, E_{i j}, F_{i j}\right] e^{-\int d \tau L}, \\
& L=L_{0}+L_{\eta}+L_{U}, \\
& L_{0}=t \sum_{\langle i j\rangle} \operatorname{tr}\left(F_{i j} E_{i j}^{\dagger}+E_{i j} F_{i j}^{\dagger}\right), \\
& L_{\eta}=\sum_{i} \eta_{i}^{\dagger}\left(\partial_{\tau} \mathbf{I}-i \vec{\Omega}_{i} \cdot \vec{\tau}\right) \eta_{i}-t \sum_{\langle i j\rangle}\left(\eta_{i}^{\dagger} F_{i j} \eta_{j}+H . c .\right), \\
& L_{U}=\frac{1}{4 u} \sum_{i} \operatorname{tr}\left(-i U_{i} \partial_{\tau} U_{i}^{\dagger}+\vec{\Omega}_{i} \cdot \vec{\tau}+i \mu U_{i} \tau_{3} U_{i}^{\dagger}\right)^{2} \\
& -t \sum_{\langle i j\rangle} \operatorname{tr}\left(U_{j}^{\dagger} E_{i j}^{\dagger} U_{i} \tau_{3}+H . c .\right),
\end{aligned}
$$

where $E_{i j}$ and $F_{i j}$ are HS matrix fields associated with hopping of $\eta_{i}$ fermions and $\phi_{k i}$ bosons, respectively.

We make an ansatz for the hopping matrix fields

$$
E_{i j} \approx E \exp \left[i \vec{a}_{i j} \cdot \vec{\tau}\right] \tau_{3}, \quad F_{i j} \approx F \exp \left[i \vec{a}_{i j} \cdot \vec{\tau}\right] \tau_{3},
$$

where $E$ and $F$ are longitudinal modes (amplitudes) of the hopping parameters, and $\vec{a}_{i j}$ their transverse modes (phase fluctuations), considered to be spatial components of $\mathrm{SU}(2)$ gauge fields. The reason why we introduce the $\tau_{3}$ matrix is that the fermion sector $L_{\eta}$ should recover the original electron Lagrangian Eq. (3) as the slaverotor representation[1, 10] does. This will be addressed more below Eq. (12).

Inserting Eq. (8) into Eq. (7), we obtain an effective $\mathrm{SU}(2)$ gauge theory of the Hubbard model for the Mott- 
Hubbard transition

$$
\begin{aligned}
& Z=\int D\left[\eta_{i}, \eta_{i}^{\dagger}, U_{i}, \vec{\Omega}_{i}, \vec{a}_{i j}\right] e^{-\int d \tau L}, \\
& L=L_{\eta}+L_{U}+4 t \sum_{\langle i j\rangle} E F, \\
& L_{\eta}=\sum_{i} \eta_{i}^{\dagger}\left(\partial_{\tau} \mathbf{I}-i \vec{\Omega}_{i} \cdot \vec{\tau}\right) \eta_{i} \\
& -t F \sum_{\langle i j\rangle}\left(\eta_{i}^{\dagger} e^{i \vec{a}_{i j} \cdot \vec{\tau}} \tau_{3} \eta_{j}+H . c .\right), \\
& L_{U}=\frac{1}{4 u} \sum_{i} \operatorname{tr}\left(-i U_{i} \partial_{\tau} U_{i}^{\dagger}+\vec{\Omega}_{i} \cdot \vec{\tau}+i \mu U_{i} \tau_{3} U_{i}^{\dagger}\right)^{2} \\
& -t E \sum_{\langle i j\rangle} \operatorname{tr}\left(U_{j}^{\dagger} \tau_{3} e^{-i \vec{a}_{i j} \cdot \vec{\tau}} U_{i} \tau_{3}+H . c .\right) .
\end{aligned}
$$

In the mean field approximation ignoring $\mathrm{SU}(2)$ gauge fluctuations $\vec{a}_{i j}$, the amplitudes of the hopping parameters and the $\mathrm{SU}(2)$ pseudospin order parameters are determined by

$$
\begin{aligned}
& 4 E=\left\langle\eta_{i}^{\dagger} \tau_{3} \eta_{j}+H . c .\right\rangle, \quad 4 F=\left\langle\operatorname{tr}\left(U_{j}^{\dagger} \tau_{3} U_{i} \tau_{3}+H . c .\right)\right\rangle \\
& \vec{\Omega}_{i}=i u\left\langle\eta_{i}^{\dagger} \vec{\tau} \eta_{i}\right\rangle+\frac{1}{2}\left\langle\operatorname{tr}\left[\vec{\tau}\left(-i U_{i} \partial_{\tau} U_{i}^{\dagger}+i \mu U_{i} \tau_{3} U_{i}^{\dagger}\right)\right]\right\rangle(10)
\end{aligned}
$$

If one considers an easy-axis limit $\vec{\Omega}_{i} \cdot \vec{\tau} \equiv \varphi_{i} \tau_{3}, U_{i} \equiv$ $\exp \left[i \phi_{3 i} \tau_{3}\right]$, and $\vec{a}_{i j} \cdot \vec{\tau} \equiv a_{3 i j} \tau_{3}$, Eq. (9) is reduced to the effective $\mathrm{U}(1)$ gauge Lagrangian in the slave-rotor representation

$$
\begin{aligned}
& L_{\eta}=\sum_{i} \eta_{i}^{\dagger}\left(\partial_{\tau} \mathbf{I}-i \varphi_{i} \tau_{3}\right) \eta_{i} \\
& -t F \sum_{\langle i j\rangle}\left(\eta_{i}^{\dagger} e^{i a_{3 i j} \tau_{3}} \tau_{3} \eta_{j}+H . c .\right), \\
& L_{U}=\frac{1}{2 u} \sum_{i}\left(\partial_{\tau} \phi_{3 i}-\varphi_{i}-i \mu\right)^{2} \\
& -2 t E \sum_{\langle i j\rangle} \cos \left(\phi_{3 j}-\phi_{3 i}-a_{3 i j}\right) .
\end{aligned}
$$

One can perform a mean field analysis[1] to show that there exists a coherent-incoherent transition of $\phi_{3 i}$ fields at half filling $\left(\varphi_{i}=\mu=0\right)$, identified with the MottHubbard transition from a spin liquid Mott insulator to a Fermi liquid metal.

On the other hand, considering an easy-plane limit $\vec{\Omega}_{i}$. $\vec{\tau} \equiv \Phi_{i}^{R} \tau_{1}, U_{i} \equiv \exp \left[i \phi_{1 i} \tau_{1}\right]$, and $\vec{a}_{i j} \cdot \vec{\tau} \equiv a_{1 i j} \tau_{1}$, we find another effective $\mathrm{U}(1)$ gauge Lagrangian[11]

$$
\begin{aligned}
& L_{\eta}=\sum_{i} \eta_{i}^{\dagger}\left(\partial_{\tau} \mathbf{I}-i \Phi_{i}^{R} \tau_{1}\right) \eta_{i} \\
& -t F \sum_{\langle i j\rangle}\left(\eta_{i}^{\dagger} e^{i a_{1 i j} \tau_{1}} \tau_{3} \eta_{j}+H . c .\right), \\
& L_{U}=\frac{1}{2 u} \sum_{i}\left(\partial_{\tau} \phi_{1 i}-\Phi_{i}^{R}\right)^{2} \\
& -2 t E \sum_{\langle i j\rangle} \cos \left(\phi_{1 j}+\phi_{1 i}-a_{1 i j}\right) .
\end{aligned}
$$

We note that Eq. (12) can be reduced to Eq. (3) with $\vec{\Omega}_{i} \cdot \vec{\tau} \equiv \Phi_{i}^{R} \tau_{1}$ after the gauge transformations of $\Phi_{i}^{R} \rightarrow \Phi_{i}^{R}+\partial_{\tau} \phi_{1 i}, a_{1 i j} \rightarrow a_{1 i j}+\phi_{1 i}+\phi_{1 j}$, and Eq. (4) without $\phi_{2 i}$ and $\phi_{3 i}$ are utilized. If the $\tau_{3}$ matrix is not utilized in Eq. (8), the hopping term in $L_{U}$ vanishes, and Eq. (3) cannot be recovered from Eq. (12). Ignoring gauge fluctuations, and replacing $\Phi_{i}^{R}$ with $\Phi_{0}$ as the mean field approximation, we obtain the superconducting order parameter given by $\Phi_{0}=i u\left\langle\eta_{i}^{\dagger} \tau_{1} \eta_{i}\right\rangle$. It turns out to be zero because double occupancy costs too much energy. Thus, there is no phase transition in the fermion Lagrangian as the case of the easy-axis limit.

To examine the boson Lagrangian in the mean field level, we resort to large $N$ generalization, following the same method in the slave-rotor representation.[1] Introducing the $N$-component rotor field $Y_{i}$, we rewrite $L_{U}$ in Eq. (12) as

$$
\begin{aligned}
& L_{Y}=\frac{1}{2 u} \sum_{i}\left(\partial_{\tau} Y_{i}^{\dagger}\right)\left(\partial_{\tau} Y_{i}\right)-t E \sum_{\langle i j\rangle}\left(Y_{i}^{\dagger} Y_{j}^{\dagger}+Y_{j} Y_{i}\right) \\
& -i \sum_{i} \lambda_{i}\left(\left|Y_{i}\right|^{2}-1\right),
\end{aligned}
$$

where $\lambda_{i}$ is a Lagrange multiplier field for the rotor constraint. If we represent Eq. (13) in terms of $Y_{i}=R_{i}+i I_{i}$ and $Y_{i}^{\dagger}=R_{i}-i I_{i}$, we find the mean field action of collective pair excitations

$$
\begin{aligned}
& S_{M F}=\int_{0}^{\beta} d \tau\left[\frac{1}{2 u} \sum_{i}\left[\left(\partial_{\tau} R_{i}\right)^{2}+\left(\partial_{\tau} I_{i}\right)^{2}\right]\right. \\
& \left.-2 t E \sum_{\langle i j\rangle}\left(R_{i} R_{j}+I_{i} I_{j}\right)-i \lambda_{i} \sum_{i}\left(R_{i}^{2}+I_{i}^{2}-1\right)\right](14)
\end{aligned}
$$

One can find that Eq. (14) is exactly the same as $\int_{0}^{\beta} d \tau L_{U}$ in Eq. (11) at half filling in the mean field level if the large $N$ generalization for $L_{U}$ in Eq. (11) is performed by introducing the rotor field $X_{i}=R_{i}+i I_{i}$. Thus, the mean field analysis in Eq. (11) can be directly applied to Eq. (12).[12] This leads us to conclude that both $\phi_{3 i}$ and $\phi_{1 i}$ fields are simultaneously incoherent in $(u / t)>(u / t)_{0}$, and coherent in $(u / t)<(u / t)_{0}$ at half filling, where $(u / t)_{0}$ is the critical value for the Mott transition.[1] Fundamentally, the reason why both fields should be coherent simultaneously at half filling is the presence of the $\mathrm{SU}(2)$ symmetry at half filling. The slave-rotor action should be symmetric (invariant) under the transformation $\phi_{1 i} \longleftrightarrow \phi_{3 i}$ at half filling in the mean field approximation.

However, if we consider the case away from half filling, we can obtain a different result. In the zero doping limit we take into account the following effective rotor actions from Eq. (9) in the mean field approximation

$$
\begin{aligned}
& L_{C R}=\frac{1}{2 u} \sum_{i}\left(\partial_{\tau} \phi_{3 i}-i \mu\right)^{2}-2 t E \sum_{\langle i j\rangle} \cos \left(\phi_{3 j}-\phi_{3 i}\right), \\
& L_{P R}=\frac{1}{2 u} \sum_{i}\left(\partial_{\tau} \phi_{1 i}\right)^{2}-2 t E \sum_{\langle i j\rangle} \cos \left(\phi_{1 j}+\phi_{1 i}\right) . \quad(15)
\end{aligned}
$$




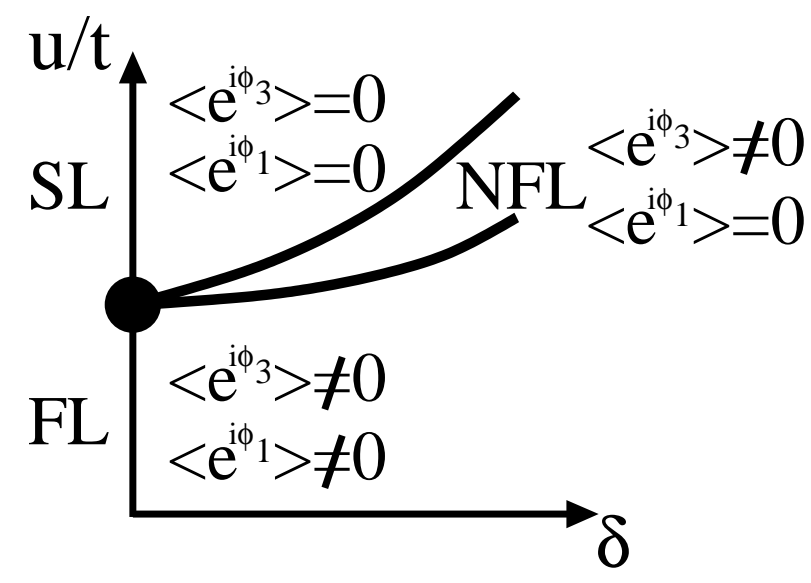

FIG. 1: A schematic phase diagram in the $\mathrm{SU}(2)$ gauge theory of the Hubbard model near half filling

It is important to see that the chemical potential plays the role of Berry phase in the charge-rotor field $e^{i \phi_{3 i}}$, given by $\mu=u \delta$.[10] One may understand this as $\mathrm{SU}(2)$ symmetry breaking due to hole doping. The presence of the $\mathrm{SU}(2)$ symmetry breaking field is expected to allow an intermediate phase, where only one phase field is condensed. It is well known that the Berry phase acts as an effective magnetic field for vortices of $e^{i \phi_{3 i}}$ bosons in the dual representation, disturbing their condensation.[13] This implies that condensation of $e^{i \phi_{3 i}}$ bosons can survive to $(u / t)_{\delta}$ larger than the $(u / t)_{0}$ of half filling. As a result, the phase characterized by $\left\langle e^{i \phi_{3 i}}\right\rangle \neq 0$ and $\left\langle e^{i \phi_{1 i}}\right\rangle=0$ is expected to appear in the parameter range of $(u / t)_{0}<(u / t)<(u / t)_{\delta}$.

A possible phase diagram in the $\mathrm{SU}(2)$ gauge theory of the Hubbard model is shown in Fig. 1, ignoring antiferromagnetic long range order. Here SL, FL, and NFL represent spin liquid, Fermi liquid, and non-Fermi liquid, respectively. It should be noted that the present mean field analysis is applicable near half filling because the doping dependence in the hopping amplitudes $E$ and $F$ is not fully accounted in our analysis. In the small $u / t$ limit both the $\phi_{3 i}$ and $\phi_{1 i}$ bosons would be condensed, resulting in a conventional Fermi liquid metal. In the large $u / t$ limit the two bosons would be gapped, thus a spin liquid Mott insulator is expected to arise since charge fluctuations are gapped, but spin excitations remain massless. On the other hand, in the intermediate range of $u / t$ only the $\phi_{3 i}$ bosons can be condensed while the $\phi_{1 i}$ bosons are not as a result of the Berry phase contribution ( $\mathrm{SU}(2)$ symmetry breaking) for the $\phi_{3 i}$ field due to hole doping.

The intermediate phase is quite interesting in the sense that although it is a metallic phase, there is no coherent electron-like quasiparticle peak near the Fermi energy. If one introduces an external electromagnetic field $A_{i j}$ in Eq. (9), he can find the following expression of the effective $\mathrm{SU}(2)$ gauge theory in the presence of an elec- tromagnetic field

$$
\begin{aligned}
& L_{\eta}=\sum_{i} \eta_{i}^{\dagger}\left(\partial_{\tau} \mathbf{I}-i \vec{\Omega}_{i} \cdot \vec{\tau}\right) \eta_{i} \\
& -t F \sum_{i j} \eta_{i}^{\dagger} e^{i \vec{a}_{i j} \cdot \vec{\tau}} \tau_{3} e^{i A_{i j} \tau_{3}} \eta_{j}, \\
& L_{U}=\frac{1}{4 u} \sum_{i} \operatorname{tr}\left(-i U_{i} \partial_{\tau} U_{i}^{\dagger}+\vec{\Omega}_{i} \cdot \vec{\tau}+i \mu U_{i} \tau_{3} U_{i}^{\dagger}\right)^{2} \\
& -t E \sum_{i j} \operatorname{tr}\left(U_{j}^{\dagger} e^{-i A_{i j} \tau_{3}} \tau_{3} e^{-i \vec{a}_{i j} \cdot \vec{\tau}} U_{i} \tau_{3} e^{i A_{i j} \tau_{3}}\right) .
\end{aligned}
$$

One can check that this gauge action preserves an electromagnetic $\mathrm{U}(1)$ gauge symmetry under the following $\mathrm{U}(1)$ gauge transformations

$$
\begin{aligned}
& \eta_{i} \rightarrow e^{i \vartheta_{i} \tau_{3}} \eta_{i}, \quad U_{i} \rightarrow e^{i \vartheta_{i} \tau_{3}} U_{i} e^{-i \vartheta_{i} \tau_{3}}, \\
& e^{i \vec{a}_{i j} \vec{\tau}} \rightarrow e^{i \vartheta_{i} \tau_{3}} e^{i \vec{a}_{i j} \vec{\tau}} e^{-i \vartheta_{i} \tau_{3}}, \vec{\Omega}_{i} \cdot \vec{\tau} \rightarrow e^{i \vartheta_{i} \tau_{3}} \vec{\Omega}_{i} \cdot \vec{\tau} e^{-i \vartheta_{i} \tau_{3}}, \\
& A_{i j} \rightarrow A_{i j}-\left(\vartheta_{i}-\vartheta_{j}\right),
\end{aligned}
$$

where $\vartheta_{i}$ is a $\mathrm{U}(1)$ phase angle, assumed to be independent of time for simplicity. The first line in Eq. (17) results from the condition $\psi_{i} \rightarrow e^{i \vartheta_{i} \tau_{3}} \psi_{i}$ in Eq. (4). In the mean field phase of $\left\langle e^{i \phi_{3 i}}\right\rangle \neq 0$ and $\left\langle e^{i \phi_{1 i}}\right\rangle=0$ with $\vec{\Omega}_{i}=0$ and $\vec{a}_{i j}=0$, we obtain an effective Lagrangian for the $\eta_{i}$ fermions from Eq. (16)

$$
L_{\eta}=\sum_{i} \eta_{i}^{\dagger} \partial_{\tau} \eta_{i}-t F \sum_{i j} \eta_{i}^{\dagger} e^{i A_{i j} \tau_{3}} \tau_{3} \eta_{j}
$$

This mean field action clearly exhibits a metallic behavior of the $\eta_{i}$ fermions as the Fermi liquid.[14] If one ignores the existence of the $\phi_{1 i}$ bosons, this phase is nothing but the Fermi liquid metal in the slave-rotor theory. However, there exists an important difference between this metallic phase and Fermi liquid owing to the gapped $\phi_{1 i}$ bosons; there are no electron-like quasiparticles in the present metallic phase.

One can obtain the following expression for the electron propagator in our composite field representation

$$
\begin{aligned}
& G_{e l \uparrow \uparrow}\left(i j, \tau \tau^{\prime}\right)=\left\langle T_{\tau}\left[c_{\uparrow i \tau} c_{\uparrow j \tau^{\prime}}^{\dagger}\right]\right\rangle \\
& =\left\langle T_{\tau}\left[e^{-i \phi_{1 i \tau} \tau_{1}} \eta_{i \tau} \eta_{j \tau^{\prime}}^{\dagger} e^{i \phi_{1 j \tau^{\prime}} \tau_{1}}\right]_{11}\right\rangle \\
& =\left\langle T_{\tau}\left[\cos \phi_{1 i \tau} \cos \phi_{1 j \tau^{\prime}} \eta_{i \tau+} \eta_{j \tau^{\prime}+}^{\dagger}\right]\right\rangle \\
& +\left\langle T_{\tau}\left[\sin \phi_{1 i \tau} \sin \phi_{1 j \tau^{\prime}} \eta_{i \tau-}^{\dagger} \eta_{j \tau^{\prime}-}\right]\right\rangle
\end{aligned}
$$

Using the large $N$ generalization in Eq. (14), and performing the mean field approximation, the electron propagator is expressed as the convolution of $\eta_{i}$ and $\phi_{1 i}$ propagators

$$
\begin{aligned}
& G_{e l \uparrow \uparrow}\left(i j, \tau \tau^{\prime}\right) \\
& \approx\left\langle T_{\tau}\left[R_{i \tau} R_{j \tau^{\prime}} \eta_{i \tau+} \eta_{j \tau^{\prime}+}^{\dagger}\right]\right\rangle+\left\langle T_{\tau}\left[I_{i \tau} I_{j \tau^{\prime}} \eta_{i \tau-}^{\dagger} \eta_{j \tau^{\prime}-}\right]\right\rangle \\
& =\left\langle T_{\tau}\left[R_{i \tau} R_{j \tau^{\prime}}\right]\right\rangle\left(\left\langle T_{\tau}\left[\eta_{i \tau+} \eta_{j \tau^{\prime}+}^{\dagger}\right]\right\rangle+\left\langle T_{\tau}\left[\eta_{i \tau-}^{\dagger} \eta_{j \tau^{\prime}-}\right]\right\rangle\right) .
\end{aligned}
$$


Interestingly, this structure is essentially the same as that of the electron green's function in the slave-rotor representation.[15] Since there is a gap in the boson spectrum, the electron spectral function shows no sharp peak at zero energy[1] in the metallic phase, implying a pseudogap in electron excitations. Although there exists a pseudogap in the electron spectrum, this phase is identified with an anomalous metal because charge carriers are not electrons but fractionalized electrons as shown in Eq. (18).[14]

One cautious person may suspect the stability of this metallic phase against instanton excitations of compact $\mathrm{U}(1)$ gauge fields beyond the mean field approximation.[16, 17] The effective $\mathrm{U}(1)$ gauge Lagrangian for the non-Fermi liquid metal can be considered as

$$
\begin{aligned}
& L_{e f f} \approx \sum_{i} \eta_{i}^{\dagger}\left(\partial_{\tau} \mathbf{I}-i a_{1 i \tau} \tau_{1}\right) \eta_{i} \\
& -t F \sum_{i j} \eta_{i}^{\dagger} e^{i a_{1 i j} \tau_{1}} \tau_{3} \eta_{j}-\frac{1}{g^{2}} \sum_{\square} \cos \left(\partial \times a_{1}\right),
\end{aligned}
$$

where $g$ is an internal gauge charge of the $\eta_{i}$ fermions. Condensation of the $\phi_{3 i}$ bosons would result in massive gauge fluctuations $a_{3 i j}$ (Anderson-Higgs mechanism), thus ignored in the low energy limit. It was argued that if $\tau_{1}$ in the gauge coupling is replaced with $\tau_{3}$, the effective U(1) gauge Lagrangian lies in a critical phase identified with a renormalization group fixed point, and can be stable against instanton excitations due to critical matter fields.[17] However, in the present case the stability of the anomalous metallic phase is not clearly guaranteed yet because screening of an internal gauge charge appears in the particle-particle channel instead of the particle-hole channel shown in the previous study,[17] thus the structure of renormalization group equations can be totally different from those in the previous study.
The stability of this non-Fermi liquid metal against disorder should be also answered for this phase to be a genuine metallic phase. The present author investigated the role of disorder in the two dimensional fermion system with long range gauge interactions, where $\tau_{1}$ in the gauge coupling is replaced with $\tau_{3}$ like the above. $[6,18]$ In this case the long range interactions are shown to make the fermion system stable against weak disorder even in two dimensions because the gauge interactions let the fermions lie in a critical phase. Remember that criticality can protect fermions from localization due to disorder.[5, 6, 18] However, the present case should be addressed more clearly because of difference of the gauge couplings.

In this paper we proposed that an anomalous metallic phase can result from local pairing fluctuations near the Mott critical point. We extended the $\mathrm{U}(1)$ gauge theory of the Hubbard model $[1,2,10]$ by allowing not only density fluctuations but also local pairing excitations, and derived an $\mathrm{SU}(2)$ gauge theory in terms of collective density and pairing fluctuations with gapless fermion excitations. We showed that there exists an interesting intermediate phase between the spin liquid Mott insulator and Fermi liquid metal, characterized by softening of local charge fluctuations as the Fermi liquid, but gapping of local pairing excitations. This behavior of collective bosons can be allowed from the Berry phase contribution for charge fluctuations due to hole doping, associated with $\mathrm{SU}(2)$ symmetry breaking. The intermediate phase turns out to be a metallic state without electron-like quasiparticles, thus identified with an anomalous nonFermi liquid metal. Role of gauge fluctuations should be addressed near future.

K.-S. Kim thanks Drs. J.-H. Han, A. Tanaka, M. Kohno, Y. Nonomura, X. Hu, Q. Li, and A. Furusaki for helpful discussions.
[1] S. Florens and A. Georges, Phys. Rev. B 70, 035114 (2004).

[2] S.-S. Lee and P. A. Lee, Phys. Rev. Lett. 95, 036403 (2005).

[3] E. Abrahams, S. V. Kravchenko, and M. P. Sarachik, Rev. Mod. Phys. 73, 251 (2001).

[4] A. Kapitulnik, N. Mason, S. A. Kivelson, and S. Chakravarty, Phys. Rev. B 63, 125322 (2001).

[5] S. Chakravarty, L. Yin, and E. Abrahams, Phys. Rev. B 58, R559 (1998).

[6] Ki-Seok Kim, Phys. Rev. B 72, 014406 (2005); Phys. Rev. B 70, 140405(R) (2004).

[7] P. Phillips and D. Dalidovich, Phys. Rev. B 65, 081101 (2002); R. L. Jack and D. K. K. Lee, Phys. Rev. B 66, 104526 (2002); D. Das and S. Doniach, Phys. Rev. B 60, 1261 (1999).

[8] D. Belitz and T. R. Kirkpatrick, Phys. Rev. B 56, 6513 (1997); D. Belitz, T. R. Kirkpatrick, and T. Vojta, Phys. Rev. B 65, 165112 (2002); A. Chubukov, C. Pepin, and
J. Rech, Phys. Rev. Lett. 92, 147003 (2004); Ar. Abanov and A. Chubukov, Phys. Rev. Lett. 93, 255702 (2004).

[9] The present decomposition scheme looks somewhat similar to the slave-boson representation of the $t-J$ model. However, our decomposition scheme is nothing to do with the slave-boson representation because the slave-boson representation originates from solving the single occupancy constraint in the $t-J$ model while the present decomposition does not. It should be noted that this decomposition is not arbitrary since the collective mode $U_{\sigma \sigma^{\prime}}$ is determined by its HS field, as will be discussed in the text.

[10] Ki-Seok Kim, cond-mat/0510564.

[11] One can derive the pair-rotor theory Eq. (12) in the canonical quantization method by decomposing the electron Hilbert space into the composite Hilbert space of $|\psi\rangle=|\eta\rangle \otimes\left|\Delta^{R}\right\rangle$ based on the composite field representation $\psi_{i}=e^{-i \phi_{1 i} \tau_{1}} \eta_{i}$, where $\Delta^{R}$ represents the density of an electron pair. This enlarged composite Hilbert space 
is reduced to the original electron one by the constraint $\Delta_{i}^{R}=\psi_{i}^{\dagger} \tau_{1} \psi_{i}$. This canonical quantization can be realized in the following path integral expression

$$
\begin{aligned}
& Z=\int\left[D \eta_{i}, \phi_{1 i}, \Phi_{i}^{R}, \Delta_{i}^{R}\right] \exp \left[-\int d \tau\left\{\sum_{i} \eta_{i}^{\dagger} \partial_{\tau} \eta_{i}\right.\right. \\
& -t \sum_{i j} \eta_{i}^{\dagger} e^{i \phi_{1 i} \tau_{1}} \tau_{3} e^{-i \phi_{1 j} \tau_{1}} \eta_{j} \\
& \left.\left.+\sum_{i}\left(u \Delta_{i}^{R 2}-i \Delta_{i}^{R} \partial_{\tau} \phi_{1 i}+i \Phi_{i}^{R}\left(\Delta_{i}^{R}-\psi_{i}^{\dagger} \tau_{1} \psi_{i}\right)\right)\right\}\right]
\end{aligned}
$$

resulting in the pair-rotor theory Eq. (12) after integrating out the $\Delta_{i}^{R}$ field, and performing the HS transformation for the hopping term. The term $-i \Delta_{i}^{R} \partial_{\tau} \phi_{1 i}$ leads us to identify the $e^{-i \phi_{1 i} \tau_{1}}$ operator with an annihilation operator of an electron pair.

[12] Condensation of the $\phi_{1 i}$ bosons $\left(R_{i}\right.$ and $\left.I_{i}\right)$ is determined by the following self-consistent equations

$$
\begin{aligned}
& 1=\int_{-D}^{D} d \epsilon D(\epsilon) \frac{1}{\beta} \sum_{\omega_{n}} \frac{1}{\omega_{n}^{2} / u+E \epsilon+\lambda}, \\
& D F=-\int_{-D}^{D} d \epsilon D(\epsilon) \frac{1}{\beta} \sum_{\omega_{n}} \frac{\epsilon}{\omega_{n}^{2} / u+E \epsilon+\lambda}, \\
& D E=-2 \int_{-D}^{D} d \epsilon D(\epsilon) \epsilon n_{F}(F \epsilon),
\end{aligned}
$$

where $D$ is the half bandwidth, $D(\epsilon)$ the band density of states, and $n_{F}(\epsilon)$ the Fermi-Dirac distribution function. Here $-i \lambda_{i}$ is replaced with $\lambda$. Solving the above equations with the density of states $D(\epsilon)=1 /(2 D)$ gives at zero temperature

$$
\begin{aligned}
& \lambda=\frac{u}{2}+\frac{D^{2}}{8 u}, \\
& F=\frac{4 u^{2}+D^{2}}{16 D^{2} u}\left(2 u+D-\frac{5}{3}|2 u-D|\right), \\
& E=\frac{1}{2} .
\end{aligned}
$$

Condensation of the $\phi_{1 i}$ bosons occurs when $\lambda-E D=0$. These equations are exactly the same as those for the $\phi_{3 i}$ bosons as a result of the $\mathrm{SU}(2)$ symmetry.

[13] This is exactly the same phenomenon as the superconducting transition temperature decreases under external magnetic fields since the condensation amplitude becomes weak due to external magnetic fields.
The Ginzburg-Landau functional under external magnetic fields is given by

$$
S_{G L}=\int d^{2} x\left[|(\nabla-i \mathbf{A}) \Phi|^{2}+V(|\Phi|)+\frac{1}{8 \pi}|\nabla \times \mathbf{A}|^{2}\right],
$$

where $\Phi$ is a boson field, $V(|\Phi|)$ its effective potential, and $\mathbf{A}$ an electromagnetic field. In the present problem $\Phi$ corresponds to a $\phi_{3}$ vortex field, and the external magnetic field the chemical potential, i.e., $\nabla \times \mathbf{A}=\mu \mathbf{z}$. This functional is well analyzed in the textbook, M. Tinkham, Introduction to Superconductivity (2nd edition), Chs. 4 and 5 (McGraw-Hill, Inc., 1996). One can find that the presence of magnetic fields weakens the condensation amplitude as $|\Phi|^{2} \approx 1-$ const. $\cdot H^{2}$, where $H$ is an external magnetic field, here $H=\mu=u \delta$.

[14] It is important to realize that the dc conductivity $\sigma_{e l}$ is given by only the fermion contribution $\sigma_{e l}=\sigma_{\eta}$ instead of the Ioffe-Larkin form [L. B. Ioffe and A. I. Larkin, Phys. Rev. B 39, 8988 (1989)] $\sigma_{e l}=\sigma_{\phi_{1}} \sigma_{\eta} /\left(\sigma_{\phi_{1}}+\sigma_{\eta}\right)$, where $\sigma_{\phi_{1}}\left(\sigma_{\eta}\right)$ is the $\phi_{1 i}\left(\eta_{i}\right)$ conductivity, even if $\mathrm{U}(1)$ gauge fluctuations $a_{1 \mu}$ are allowed at the gaussian order. Note that the $a_{3 \mu}$ fluctuations are massive owing to the Anderson-Higgs mechanism, as discussed in the text. $\sigma_{e l}=\sigma_{\eta}$ originates from the fact that there are $\tau_{1}$ and $\tau_{3}$ matrices in the gauge couplings with $a_{1 \mu}$ and $A_{\mu}$ respectively, and the correlation function between a pair current with $\tau_{1}$ and a charge current with $\tau_{3}$ vanishes due to orthogonality between the $\tau_{1}$ and $\tau_{3}$ matrices. As a result, the conductivity is given by the fermion contribution only, identifying this phase with a metal owing to the gapless $\eta_{i}$ excitations.

[15] In the slave-rotor representation[1] the electron propagator is given by

$$
\begin{aligned}
& G_{e l \uparrow \uparrow}\left(i j, \tau \tau^{\prime}\right)=\left\langle T_{\tau}\left[c_{\uparrow i \tau} c_{\uparrow j \tau^{\prime}}^{\dagger}\right]\right\rangle \\
& =\left\langle T_{\tau}\left[e^{-i \phi_{3 i \tau} \tau_{3}} \eta_{i \tau} \eta_{j \tau^{\prime}}^{\dagger} e^{i \phi_{3 j \tau^{\prime}} \tau_{3}}\right]_{11}\right\rangle \\
& =\left\langle T_{\tau}\left[e^{-i\left(\phi_{3 i \tau}-\phi_{3 j \tau^{\prime}}\right)} \eta_{i \tau+} \eta_{j \tau^{\prime}+}^{\dagger}\right]\right\rangle \\
& +\left\langle T_{\tau}\left[e^{i\left(\phi_{3 i \tau}-\phi_{3 j \tau^{\prime}}\right)} \eta_{i \tau-}^{\dagger} \eta_{j \tau^{\prime}-}\right]\right\rangle \\
& \approx\left\langle T_{\tau}\left[e^{i\left(\phi_{3 i \tau}-\phi_{3 j \tau^{\prime}}\right)}\right]\right\rangle\left(\left\langle T_{\tau}\left[\eta_{i \tau+} \eta_{j \tau^{\prime}+}^{\dagger}\right]\right\rangle+\left\langle T_{\tau}\left[\eta_{i \tau-}^{\dagger} \eta_{j \tau^{\prime}-}\right]\right\rangle\right)
\end{aligned}
$$

[16] Ki-Seok Kim, Phys. Rev. B 72, 035109 (2005); Phys. Rev. B 72, 214401 (2005).

[17] Ki-Seok Kim, Phys. Rev. B 72, 245106 (2005).

[18] Ki-Seok Kim, Phys. Rev. B 73, 235115 (2006). 